\title{
Nernst effect and dimensionality in the quantum limit
}

\author{
Zengwei Zhu ${ }^{1,2}$, Huan Yang ${ }^{1}$, Benoît Fauquée', Yakov Kopelevich ${ }^{3}$ and Kamran Behniaa ${ }^{1 \star}$
}

The Nernst effect has recently emerged as a very sensitive, yet poorly understood, probe of electron organization in solids ${ }^{1-4}$. Graphene, a single layer of carbon atoms set in a honeycomb lattice, embeds a two-dimensional gas of massless electrons ${ }^{5}$ and hosts a particular version of the quantum Hall effect ${ }^{6,7}$. Recent experimental investigations of its thermoelectric response ${ }^{8-10}$ are in agreement with the theory conceived for a two-dimensional electron system in the quantum Hall regime ${ }^{11,12}$. Here, we report on a study of graphite $^{13}$, a macroscopic stack of graphene layers, which establishes a fundamental link between the dimensionality of an electronic system and its Nernst response. In striking contrast with the single-layer case, the Nernst signal sharply peaks whenever a Landau level meets the Fermi level. Thus, the degrees of freedom provided by finite interlayer coupling lead to an enhanced thermoelectric response in the vicinity of the quantum limit. As Landau quantization slices a threedimensional Fermi surface, each intersection of a Landau level with the Fermi level modifies the Fermi-surface topology. According to our results, the most prominent signature of such a topological phase transition emerges in the transverse thermoelectric response.

The Nernst effect is the transverse voltage generated by a longitudinal thermal gradient in the presence of a magnetic field. Our study of this effect in graphite, the first carried out at low temperatures and high magnetic fields, uncovers the crucial role of interlayer coupling in a three-dimensional (3D) metal. When the field is strong enough to push the system to the quantum limit, the quantum oscillations of the Nernst response easily dwarf the oscillations seen in other transport coefficients. Moreover, the Nernst signal sharply peaks whenever a Landau level intersects the chemical potential. Both of these features are absent in monolayer graphene $e^{8-10}$, but were previously reported in bulk bismuth ${ }^{14}$. The Nernst peaks seem to be the signatures of a topological phase transition $^{15}$, which occurs at the intersection of Landau and Fermi levels ${ }^{16}$. Such a field-induced modification of the Fermi-surface topology at lower Landau levels is exclusive to three dimensions, for which no adequate quantitative description of the thermoelectric response in the vicinity of the quantum limit is yet available. The results point to the configurational degrees of freedom associated with the finite dispersion of electrons along the magnetic field as the source of a huge off-diagonal thermoelectric response.

Figure 1 shows the thermal evolution of the field dependence of the Nernst signal, $S_{x y}$, in two highly oriented pyrolytic graphite (HOPG) samples. As the temperature decreases, quantum oscillations become sharper and their visibility extends to lower fields. In graphite, quantum oscillations of various physical properties such as resistivity (the Shubnikov-de Hass effect) ${ }^{17}$, magnetic susceptibility (the de Hass-van Alphen effect) ${ }^{18}$, the Hall coefficient and thermopower ${ }^{19}$ were measured many years ago and the results are in agreement with the structure of the Fermi surface predicted by the Slonczewski-Weiss-McClure (SWM) model ${ }^{20,21}$. More recently, quantum oscillation studies using new techniques for analysis and measurements have emerged and the degree of accuracy with which the SWM model describes the fine structure of the Fermi surface has become a subject of ongoing debate ${ }^{22,23}$ (see Supplementary Information).

The particularity of the Nernst effect as a probe of quantum oscillations at low-index Landau levels is highlighted in Fig. 2. Quantum oscillations are visible in the field dependence of both diagonal and off-diagonal components of electric and thermoelectric conductivity tensors. However, only in the case of transverse thermoelectric response, the oscillating signal exceeds by far the non-oscillating background. In contrast, the Shubnikov-de Haas oscillations represent a tiny fraction of the overall longitudinal resistivity. Moreover, whereas the longitudinal response dominates for resistivity $\left(\rho_{x x} \gg \rho_{x y}\right)$, the Nernst signal is an order of magnitude larger than the Seebeck coefficient $\left(S_{x y} \gg S_{x x}\right)$. The large magnitude of the Nernst coefficient in graphite (second only to bismuth (ref. 24) among metals) is an expected consequence of the high mobility $\left(3 \times 10^{5} \mathrm{~cm}^{2} \mathrm{~V}^{-1} \mathrm{~s}^{-1}\right)$ and the low Fermi energy $(19 \mathrm{meV})$ of the system (see Supplementary Information for details).

Figure 3 compares the fine structure of $\rho_{x y}$ and $S_{x y}$ when the lowest Landau levels cross the Fermi level. The Nernst response peaks when the magnetic field attains a value corresponding to an intersection of a Landau level with the Fermi level. The magnitudes of these fields were determined by theory ${ }^{25}$ and confirmed by experiment ${ }^{19}$ many years ago (see Supplementary Information for details on the indexing of the Landau levels and a comparison of the fine structure in $S_{x y}$ and $S_{x x}$ ). The peaks in $S_{x y}$ for the lowest Landau levels of the two pockets can be clearly associated with a jump or drop in $\rho_{x y}$ depending on the sign (hole-like or electron-like) of the carriers of the pocket. Between jumps and drops, that is when the Fermi level lies between distinct Landau levels, the Hall resistivity, although not strictly flat, shows little field dependence. Presenting the same data in a different fashion in Fig. 3c, one can see that at high fields and low temperatures, $v / T$ becomes constant between two successive peaks. In other words, in the low- $T$ high- $B$ limit, when a Landau level does not intersect the Fermi level, the Nernst response becomes both $B$-linear and $T$-linear but sharply peaks otherwise, with a singularity that becomes more pronounced with cooling. As well as the two above-mentioned HOPG samples, we also measured a natural single crystal of graphite and found very similar results (see Supplementary Information).

It is illuminating to compare these features with results very recently reported for graphene $e^{8,10}$. The first difference is the sheer

\footnotetext{
${ }^{1}$ Laboratoire Photons Et Matière (UPMC-CNRS), ESPCI, 75005 Paris, France, ${ }^{2}$ Department of Physics, Zhejiang University, Hangzhou 310027, China,

${ }^{3}$ Instituto de Fisica 'Gleb Wataghin', Universidade Estadual de Campinas, UNICAMP, 13083-970 Campinas, São Paulo, Brazil.

*e-mail: kamran.behnia@espci.fr.
} 

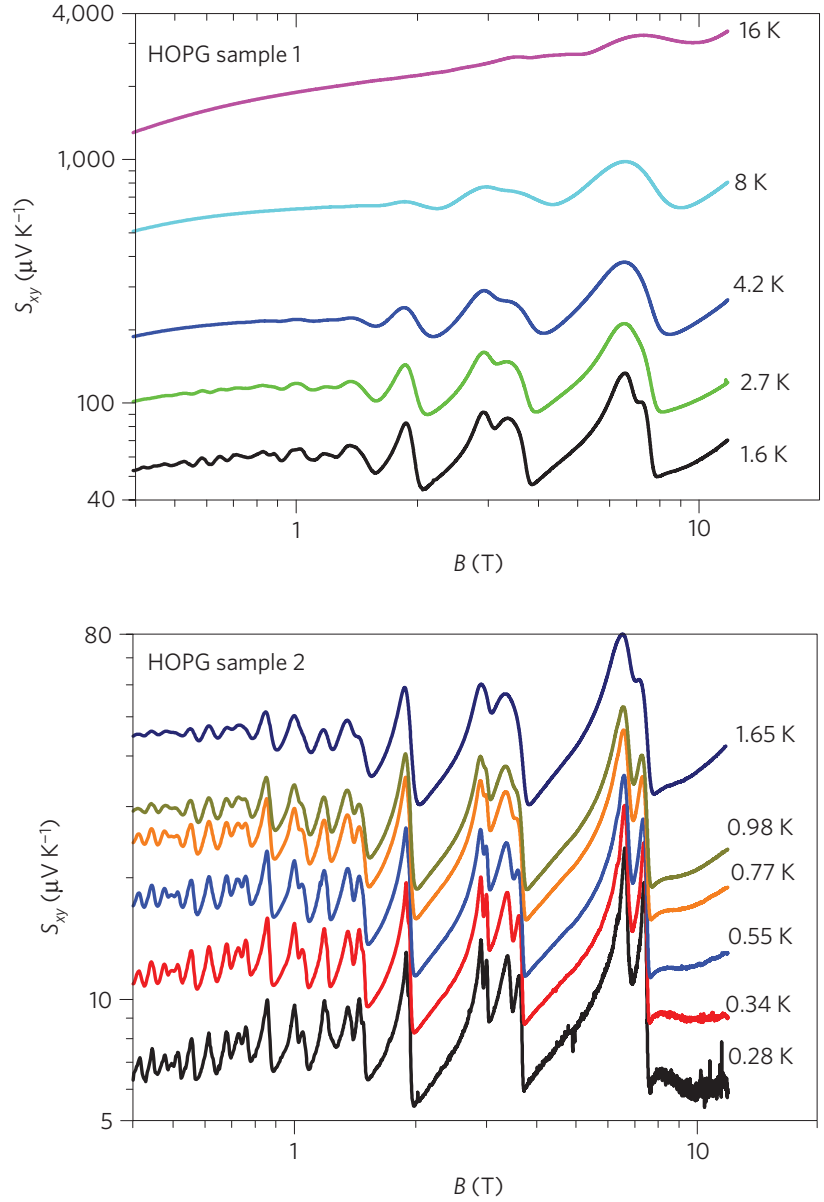

Figure 1 | The field dependence of the Nernst signal in two HOPG samples at different temperatures. The signal was measured with an in-plane thermal gradient and a field oriented perpendicular to the layers. As the temperature is lowered, oscillations become sharper and the Zeeman splitting becomes visible.

magnitude of the Nernst response. Whereas in graphene, the measurable signal at $T=10 \mathrm{~K}$ is in the range of $10-20 \mu \mathrm{V} \mathrm{K}^{-1}$, the Nernst signal in the same temperature range in graphite is two orders of magnitude larger and approaches $1 \mathrm{mV} \mathrm{K}^{-1}$ (Fig. 1, top panel). This difference is most probably due to the larger electron mobility of graphite, which can exceed by two orders of magnitude the mobility in available graphene samples (see Supplementary Information on the magnitude of the Nernst effect). The second and more fundamental difference is the field profile of the Nernst response. In graphene, the intersection of the chemical potential and a Landau level (which can be realized either by scanning the field or modifying the gate voltage) leads to a change in the sign of $S_{x y}$. In other words, the coincidence of a Landau level with the chemical potential is concomitant with a jump in $\rho_{x y}$ and a vanishing $S_{x y}$. In graphite, as seen above, the Nernst response attains its maximum in the same conditions. Note that the huge electron mobility, the origin of the large low-field non-oscillating Nernst response in graphite, can neither explain the preponderance of the oscillating component, nor its particular profile.

Interestingly, the field profile of the Nernst response we find here is analogous to the one previously reported in bulk bismuth ${ }^{14}$. On the other hand, the Nernst response of graphene ${ }^{8,10}$ presents a functional form reminiscent of the case of the $2 \mathrm{D}$ electron systems realized in semiconductor heterostructures ${ }^{26}$. In both $2 \mathrm{D}$ cases, $S_{x y}$ vanishes at the intersection of Landau and Fermi levels as predicted by the theory conceived for two dimensions ${ }^{11,12,27}$.
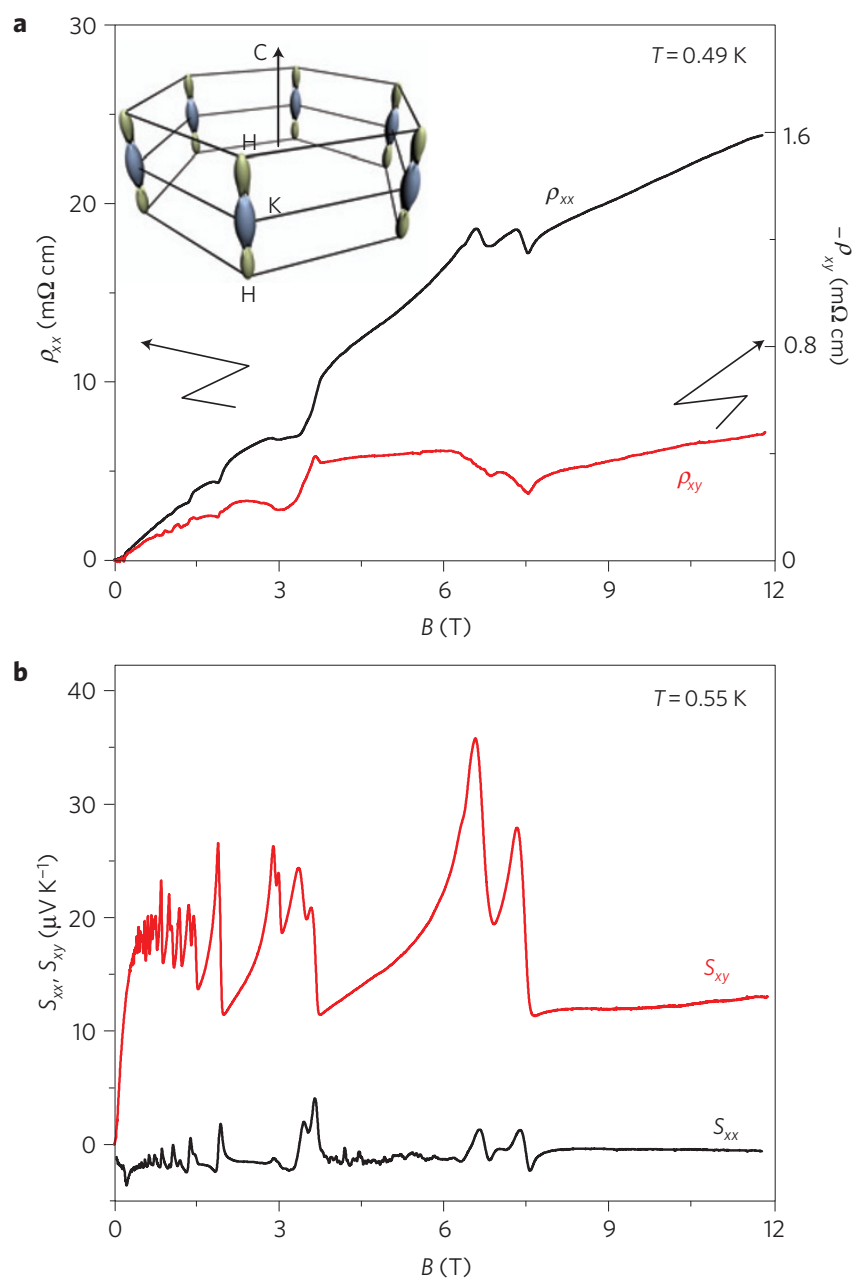

Figure 2 | Quantum oscillations of various transport coefficients at low temperatures. a, Longitudinal and Hall resistivity of sample 2. Shubnikov-de Haas oscillations superpose on a huge non-oscillating background. $\mathbf{b}$, The field dependence of the thermoelectric coefficients in the same sample. The Nernst response dominates by far the Seebeck coefficient and presents pronounced well-defined oscillations. The Fermi surface and the Brillouin zone of graphite are shown as an inset in $\mathbf{a}$. The Fermi surface consists of six pairs of adjacent ellipsoid pockets (in blue and green) hosting carriers of opposite signs.

Two features set graphene apart from both bismuth and bulk graphite. The most fundamental is the presence of a finite third-axis dispersion in the bulk materials. The second difference is that both bismuth and bulk graphite are compensated semi-metals hosting equal concentrations of mobile carriers of both signs. Experiments on graphene were carried out on a system with a single type of carrier determined by the sign of the applied gate voltage. These two features lead to the emergence of a qualitatively different transverse thermoelectric response.

According to the SWM model, the Fermi surfaces of both electrons and holes in graphite are elongated ellipsoids. The precise magnitude of interlayer coupling is a matter of debate and the Fermi surface of one type of carrier may not be closed along the $c$ axis. As a very anisotropic conductor and a bulk quasi-2D metal, HOPG has been reported to host the quantum Hall effect ${ }^{22}$.

However, a finite interlayer coupling, no matter how small, would warp the perfect cylindrical Fermi surface of a twodimensional monolayer. Its drastic consequences are shown in Fig. 4, which compares the passage of successive Landau levels through the chemical potential in the presence $(3 \mathrm{D}$, left) or absence 


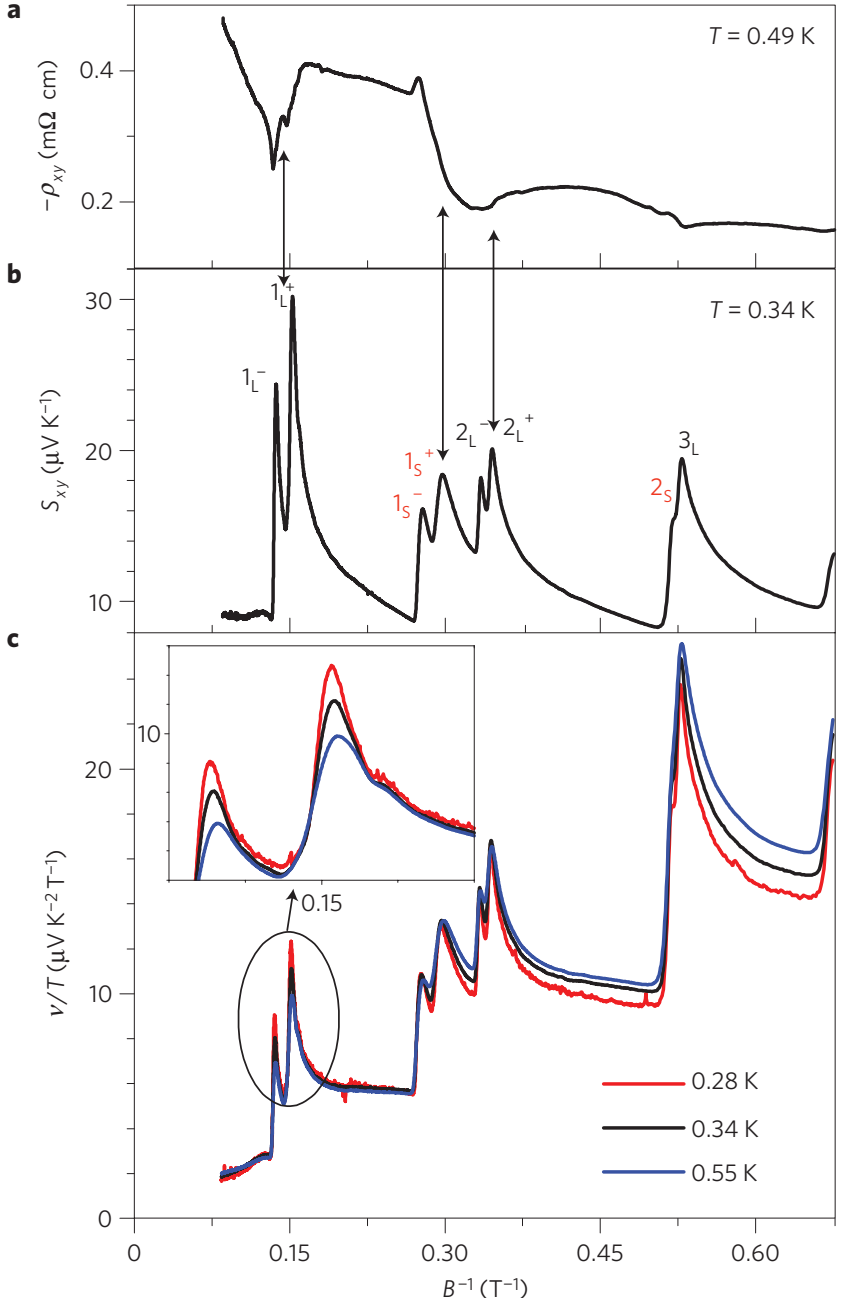

Figure $3 \mid$ Hall and Nernst effects in the vicinity of the quantum limit. a-c, The variation with inverse magnetic field of the Hall resistivity, $\rho_{x y}(\mathbf{a})$, the Nernst signal, $S_{x y}(\mathbf{b})$, and the Nernst coefficient divided by temperature, $v / T=S_{x y} / B T$, at three different temperatures (c). When a Landau level meets the Fermi level, the Hall resistivity presents a jump and the Nernst signal sharply peaks. At low temperature and high field, $S_{x y}$ becomes linear in field and temperature when the Fermi level is between two Landau levels. The inset shows a magnification of the peak positions. When a Landau level is at the Fermi level, $v / T$ increases with decreasing temperature, otherwise it attains a temperature-independent magnitude. Landau levels of the large (L) and small (S) pockets of the Fermi surface are indexed in $\mathbf{b}$.

(2D, right) of the $z$-axis dispersion. In three dimensions, Landau quantization truncates the Fermi surface. The wavevectors of mobile electrons are confined to slices formed by the intersection of the Fermi-Dirac distribution and the Landau spectrum. The thickness of these slices depends on temperature and disorder. As the field is swept, the slices move outward and downward. The Nernst peaks are concomitant with the merger of two slices. As first noticed many years ago $^{16}$, such a merger is a case of an electronic topological phase transition ${ }^{15}$. Interestingly, a singularity in the Nernst response in the vicinity of a topological transition was theoretically predicted in another context $t^{28}$. On the other hand, in the 2D case, shown on the right-hand side of the same figure, no such topological transition occurs when the ellipsoid is replaced by a perfect cylinder. Here, when the Landau level coincides with the chemical potential, the system becomes dissipative, otherwise it is gapped. a
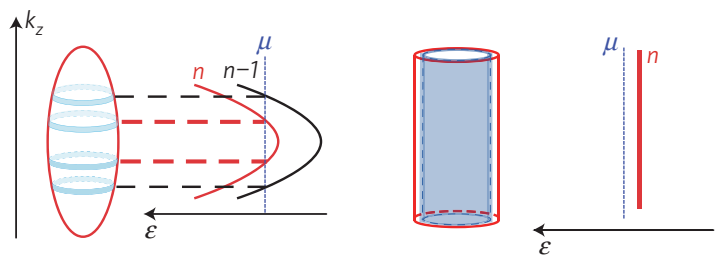

b
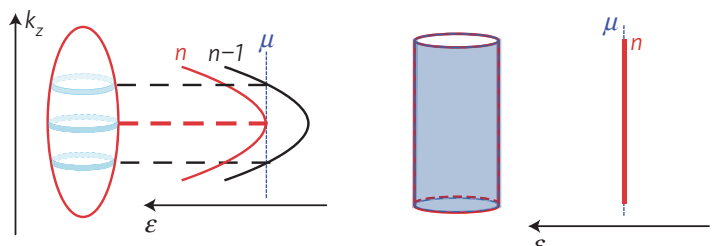

c
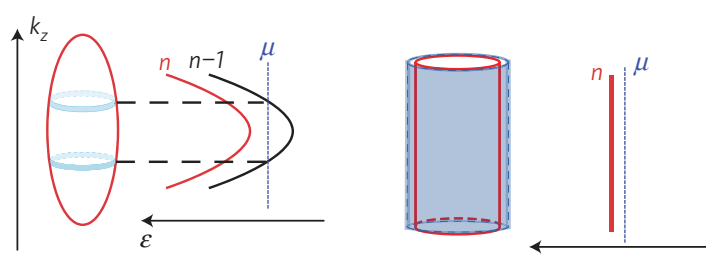

d
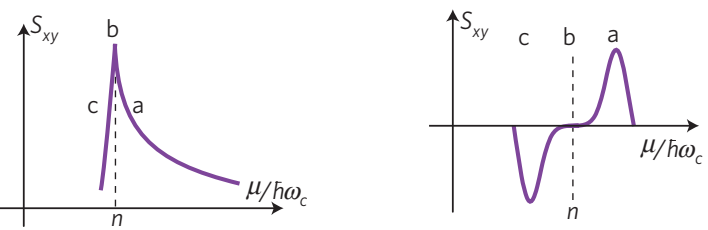

Figure 4 | Dimensionality and the profile of a Nernst quantum oscillation.

a-c, A comparison of how Landau levels (indexed $n, n-1$ ) cross the chemical potential, $\mu$, as the magnetic field is swept, in the 3D (left column) and 2D (right column) cases. In each panel, the Fermi surface with cuts of the Landau cylinders in reciprocal space is shown together with a plot of the corresponding energies. The $z$ axis is set by the direction of the applied magnetic field. In the 3D case, an ellipsoidal Fermi surface is truncated to slices, which are the intersections of the Fermi level and the Landau levels. In the 2D case, the Fermi surface is a perfect cylinder and there are no slices. d, The Nernst signal. In 3D (left panel), $S_{x y}$ peaks when the Landau level meets the Fermi level and a topological phase transition occurs by the merger of two slices. In the left column, the odd number of slices in $\mathbf{b}$ is to be contrasted with the even number of slices in $\mathbf{a}$ and $\mathbf{c}$. In 2D (right panel), $S_{x y}$ vanishes when a Landau level intersects the chemical potential. The experimentally resolved Nernst signal presents the profile in the left panel of $\mathbf{d}$ in both graphite (as reported here) and bismuth ${ }^{14}$. On the other hand, in graphene ${ }^{8-10}$ and semiconducting heterostructures ${ }^{26}$ it presents the profile in the right panel of $\mathbf{d}$.

In the $2 \mathrm{D}$ case, displacing a Landau level across the chemical potential leads to a symmetric $S_{x y}$ profile. This can be qualitatively understood in the semi-classical picture. The thermoelectric tensor $\widetilde{S}$ measures the change in conductivity tensor, $\widetilde{\sigma}$, caused by a small shift in the chemical potential:

$$
\widetilde{S}=\left.\frac{\pi^{2}}{3} \frac{k_{\mathrm{B}}^{2} T}{e} \widetilde{\sigma}^{-1} \frac{\partial \widetilde{\sigma}}{\partial \epsilon}\right|_{\epsilon=\mu}
$$

According to this equation (sometimes called the extended Mott formula), if the chemical potential happens to be at its optimal position for a maximal Hall mobility $\sigma_{x x} / \sigma_{x y}$, the transverse thermoelectric response, $S_{x y}$, is expected to vanish. This simple argument can explain the qualitative profile of the Nernst response in the $2 \mathrm{D}$ case, which is symmetric and vanishes at the intersection. 
When a Landau level is precisely at the chemical potential, any shift of the latter would diminish the Hall mobility. This zero response should be sandwiched between a positive and a negative peak (as shown in Fig. 4d, right panel). The signs of these two reflect the sign of the energy derivative when one approaches the critical field from lower fields or higher fields.

The profile of the Seebeck coefficient in the 3D graphite (see Supplementary Fig. S3) is not qualitatively different from the 2D one. On the other hand, the Nernst response in the 3D configuration emerges as a remarkable singularity. The clear asymmetry between configuration a and configuration $\mathrm{c}$ in the left panels of Fig. 4 is the key to the functional shape of the Nernst response. Experimentally, $S_{x y}$ steadily increases as the two separated slices of the Fermi surface move towards each other. It peaks when they merge to form a single one and begins a sudden drop after the Landau level moves beyond the Fermi level. The configurational entropy of the electronic states with vanishing $k_{z}$ and quantized $k_{x y}$, which emerge as this topological phase transition occurs, seems to be the source of the enhanced Nernst response exclusive to the 3D case.

The compensated nature of the bulk semi-metals under study imposes another constraint. Charge neutrality implies that any change in the density of carriers with one sign would shift the chemical potential in a way to maintain the equality between the concentration of electrons and holes. A topological transition in one (for example, hole-like) ellipsoid thus moves the chemical potential and modifies the shape of the other.

A satisfactory theoretical understanding of the Nernst effect in bulk systems across the quantum limit is still missing. It is a remarkable irony that the least theoretically understood transport coefficient happens to be the most experimentally sensitive probe of quantum oscillations. Extending these measurements to higher magnetic fields would let one probe the thermoelectric response of graphite in the extreme quantum limit. Such a study in bismuth has recently uncovered several enigmatic field scales ${ }^{29}$. Another line of investigation would be the study of $n$-layer graphene to document the details of the dimensionality cross-over found here.

Received 3 June 2009; accepted 23 September 2009; published online 8 November 2009

\section{References}

1. Wang, Y., Li, L. \& Ong, N. P. Nernst effect in high- $T_{\mathrm{c}}$ superconductors. Phys. Rev. B 73, 024510 (2006).

2. Pourret, A. et al. Observation of the Nernst signal generated by fluctuating Cooper pairs. Nature Phys. 2, 683-686 (2006).

3. Behnia, K. The Nernst effect and the boundaries of the Fermi liquid picture. J. Phys. Condens. Matter 21, 113101 (2009).

4. Cyr-Choinière, O. et al. Enhancement of the Nernst effect by stripe order in a high- $T_{\mathrm{c}}$ superconductor. Nature 458, 743-745 (2009).

5. Geim, A. K. \& Novoselov, K. S. The rise of graphene. Nature Mater. 6, 183-191 (2007).

6. Novoselov, K. S. et al. Two-dimensional gas of massless Dirac fermions in graphene. Nature 438, 197-200 (2005).

7. Zhang, Y. et al. Experimental observation of the quantum Hall effect and Berry's phase in graphene. Nature 438, 201-204 (2005).

8. Zuev, Y. M., Chang, W. \& Kim, P. Thermoelectric and magnetothermoelectric transport measurements of graphene. Phys. Rev. Lett. 102, 096807 (2009).
9. Wei, P. et al. Anomalous thermoelectric transport of Dirac particles in graphene. Phys. Rev. Lett. 102, 166808 (2009).

10. Checkelsky, J. G. \& Ong, N. P. Thermopower and Nernst effect in graphene in a magnetic field. Phys. Rev. B 80, 081413(R) (2009).

11. Jonson, M. \& Grivin, S. M. Thermoelectric effect in a weakly disordered inversion layer subject to a quantizing magnetic field. Phys. Rev. B 29, 1939-1946 (1984).

12. Oji, J. Thermomagnetic effects in two-dimensional electron systems. J. Phys. C 17, 3059-3066 (1984).

13. Brandt, N. B., Chudinovn, S. M. \& Ponomarev, Ya. G. Semimetals I. Graphite and its Compounds (Elsevier, 1988).

14. Behnia, K., Méasson, M.-A. \& Kopelevich, Y. Oscillating Nernst-Ettingshausen effect in bismuth across the quantum limit. Phys. Rev. Lett. 98, 166602 (2007).

15. Lifshitz, I. M. Anomalies of electron characteristics of a metal in the high pressure region. JETP 11, 1130-1135 (1960).

16. Blanter, Ya. M., Kaganov, M. I., Pantsulaya, A. V. \& Varlamov, A. A. The theory of electronic topological transitions. Phys. Rep. 245, 159-257 (1994).

17. Soule, D. E., McClure, J. W. \& Smith, L. B. Study of the Shubnikov-de Haas effect. Determination of the Fermi surfaces in graphite. Phys. Rev. 134, A453-A470 (1964).

18. Williamson, S. J., Foner, S. \& Dresselhaus, M. S. de Haas-van Alphen effect in pyrolytic and single-crystal graphite. Phys. Rev. 140, A1429-A1447 (1965).

19. Woollam, J. A. Graphite carrier locations and quantum transport to 10 T (100 kG). Phys. Rev. B 3, 1148-1158 (1971).

20. Slonczewski, J. C. \& Weiss, P. R. Band structure of graphite. Phys. Rev. 109, 272-279 (1958)

21. McClure, J. W. Band structure of graphite and de Haas-van Alphen effect. Phys. Rev. 108, 612-618 (1957).

22. Luk'yanchuk, I. A. \& Kopelevich, Y. Dirac and normal fermions in graphite and graphene: Implications of the quantum Hall effect. Phys. Rev. Lett. 97, 256801 (2006).

23. Schneider, J. M. et al. Consistent interpretation of the low-temperature magnetotransport in graphite using the Slonczewski-Weiss-McClure 3D band-structure calculations. Phys. Rev. Lett. 102, 166403 (2009).

24. Behnia, K., Méasson, M.-A. \& Kopelevich, Y. Nernst effect in semimetals: The effective mass and the figure of merit. Phys. Rev. Lett. 98, 076603 (2007).

25. Sugihara, K. \& Ono, S. Galvanometric properties of graphite at low temperatures. J. Phys. Soc. Jpn 21, 631-637 (1966).

26. Fletcher, R. Magnetothermoelectric effects in semiconductor systems. Semicond. Sci. Technol. 14, R1-R15 (1999).

27. Gusynin, V. P. \& Sharapov, S. G. Transport of Dirac quasiparticles in graphene: Hall and optical conductivities. Phys. Rev. B 73, 245411 (2006).

28. Livanov, D. V. Hall and Nernst effects in some models of anisotropic metals. Phys. Rev. B 60, 13439-13443 (1999).

29. Behnia, K., Balicas, L. \& Kopelevich, Y. Signatures of electron fractionalization in ultraquantum bismuth. Science 317, 1729-1731 (2007).

\section{Acknowledgements}

We thank A. Varlamov for stimulating discussions and in particular for introducing ref.16 to us. This work is supported by the Agence Nationale de Recherche (ANR-08-BLAN-0121-02) as a part of the DELICE project in France and by FAPESP and CNPq in Brazil. Z.Z. acknowledges a scholarship granted by the China Scholarship Council.

\section{Author contributions}

Z.Z. (assisted by H.Y. and B.F.) carried out the experiment and (assisted by K.B.) analysed the data and prepared the figures. Y.K. initiated this work. K.B. wrote the paper.

\section{Additional information}

The authors declare no competing financial interests. Supplementary information accompanies this paper on www.nature.com/naturephysics. Reprints and permissions information is available online at http://npg.nature.com/reprintsandpermissions. Correspondence and requests for materials should be addressed to K.B. 